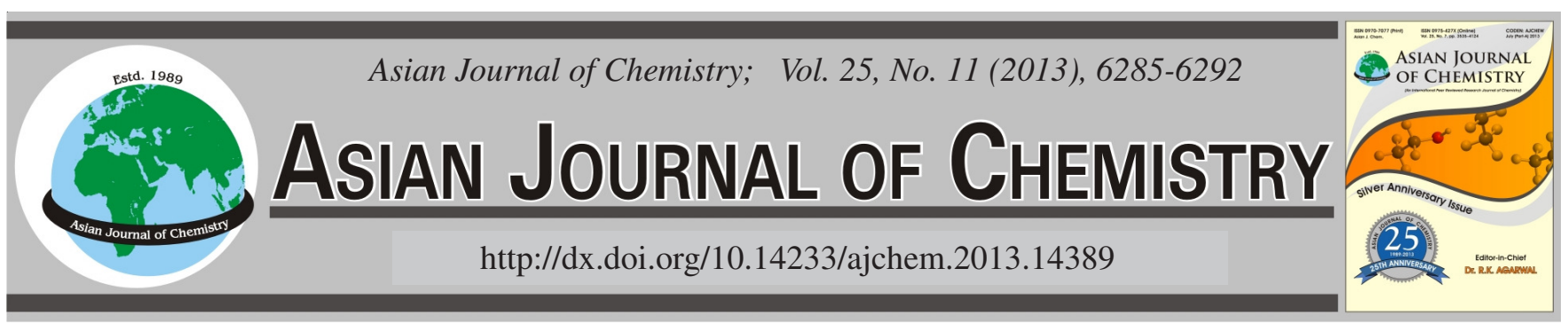

\title{
Chemical Fingerprint and Metabolic Fingerprint of Danhong Injection by HPLC-UV and HPLC-MS
}

\author{
Dan Zhou, Yanrong Zhu, Yue Guan, Wei Quan, Yuwen Li, Chao Guo, \\ Yan Weng, Jia Cui, Lei Chen, Yi Ding, Miaomiao XI and Aidong Wen*
}

Department of Pharmacy, Xijing Hospital of Fourth Military Medical University, Changle Road 17, Xi'an 710032, P.R. China

*Corresponding author: Tel/Fax: +86 29 84773636; +86 29 84775475; E-mail: adwen-2012@ hotmail.com; handsomfish@yahoo.com.cn

\begin{abstract}
Chemical fingerprint and metabolic fingerprint of Danhong injection and its raw materials (Radix Salviae miltiorrhizae and Flos carthami) were analyzed by HPLC-UV and HPLC-MS techniques. HPLC profiles, established as their characteristic fingerprints, were employed to assess the consistency and difference between Danhong injection and its raw materials. Ten batches of Danhong injections were analyzed under the same HPLC conditions. The results showed a good similarity. To determine the major constituents of Danhong injection for quality control, 19 major chromatographic peaks were characterized. Through comparison of the HPLC profiles of Danhong injection with its raw materials, it was found that they are greatly different, which indicated the changes of major constituents in the course of preparation procedure. In addition, to explore the in vivo metabolism of the major active constituents, the rat plasma was analyzed by HPLC techniques after intravenous administration of Danhong injection at different time intervals. The results indicated that danshensu, protocatechuic aldehyde, hydroxysafflor yellow A, rosmarinic acid and salvianolic acid B might be important for biological activity of Danhong injection. It could be concluded that chemical fingerprint combined with metabolic fingerprint is a useful means of controlling the quality and clarifying the possible mechanism of Danhong injection.
\end{abstract}

Key Words: Chemical fingerprint, Metabolic fingerprint, Danhong injection, Radix Salviae Miltiorrhizae, Flos carthami.

\section{INTRODUCTION}

In the development of traditional Chinese medicines (TCMs), the critical issue is the quality control. Fingerprint which shows chemical information is a method used to control quality of traditional Chinese medicine (TCM) $)^{1,2}$. In 2004, the State Food and Drug Administration (SFDA) required that all the injections made from herbal medicines and their raw materials should be standardized by chromatographic fingerprint ${ }^{3}$.

The chromatographic methods include high-performance liquid chromatographic (HPLC), gas chromatograph (GC), high performance thin layer chromatography (HPTLC) and capillary electrophoresis (CE) and they are recognized rapid, reliable and sensitive for the identification and qualification of herbal medicines ${ }^{4-7}$.

Danhong injection is a Chinese Materia Medical standardized product and made from the aqueous extracts of Radix Salviae Miltiorrhizae and Flos carthami. The water-soluble constituents in Radix Salviae Miltiorrhizae are mainly phenolic acids including danshensu, protocatechuic aldehyde, rosmarinic acid and salvianolic acid $\mathrm{B}^{8}$. Through the pharmacological and clinical research, these phenolic acids were found to be the real active constituents ${ }^{9}$. The chemical structures of phenolic acids in Danhong injection are shown in Fig. 1. Phytochemical investigation shows that safflor yellow is the main constituent in water-soluble extract of Flos carthami ${ }^{10}$. Safflor yellow consists of hydroxysafflor yellow A, safflor yellow B, safflomin A, etc. And through pharmacological and clinical investigation, hydroxysafflor yellow A is the main active component of Flos carthami ${ }^{11}$. The chemical structures and molecular weight of constituents in Danhong injection from Flos carthami are shown in Fig. 1. Danhong injection can promote blood circulation and resolve stasis and even remove stasis to promote regeneration. It is widely used to treat angina, myocardial infarction, coronary heart disease and cerebrovascular diseases ${ }^{12}$.

Although the bright prospects clinical application of Danhong injection, only danshensu, protocatechuic aldehyde and total flavonoids were quantified and used as marker for the quality control of preparation ${ }^{13}$. Hence, this existed quality control standard could not reflect the real and comprehensive active constituents of Danhong injection and therefore inadequate to control the quality of Danhong injection. So it 
<smiles>O=C(O)C(O)Cc1ccc(O)c(O)c1</smiles><smiles>O=C(O)/C=C/c1ccc(O)c(O)c1</smiles><smiles>O=Cc1ccc(O)c(O)c1</smiles>

protocateuchuic aldehyde<smiles>[R]O[R]O[R]</smiles>

salvianolic acid $B, R_{1}=R_{2}=X \quad$ Salvianolic acid $A, R=X$ linthospermic acid, $\mathrm{R}_{1}=\mathrm{X}, \mathrm{R}_{2}=\mathrm{H}$<smiles>[R2]OC(=O)/C=C/c1ccc(O[R20])c(O[R16])c1[R2]</smiles>

Salvianolic acid D, $\mathrm{R}_{1}=\mathrm{X}, \mathrm{R}_{2}=\mathrm{CH}_{2} \mathrm{COOH}, \mathrm{R}_{3}=\mathrm{R}_{4}=\mathrm{H}$ Salvianolic acid $\mathrm{H}, \mathrm{R}_{1}=\mathrm{X}, \mathrm{R}_{2}=\mathrm{R}_{4}=\mathrm{C}, \mathrm{R}_{3}=\mathrm{Y}$<smiles>CCCC1(C)CC(=O)C=C(C)C1(O)/C=C/C(C)OC(C)=O</smiles><smiles>[R]c1c(O)c([R2])c2c(c1O)C(=O)CC(c1ccc(O)cc1)O2</smiles><smiles>O=C(/C=C/c1ccc(O)cc1)C1=C(O)C2([C@H]3O[C@H](O)[C@@H](O)[C@H](CO)[C@H]3O)C(O)=C([C@@H]3O[C@H](CO)[C@@H](O)[C@H](O)[C@H]3O)C(=O)C2=C1O</smiles>

Hydroxyl of safflower yellow A

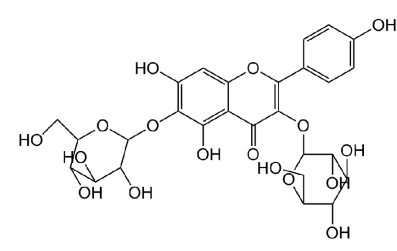

6-hydroxyl kaempferol-3,6-di-glucoside

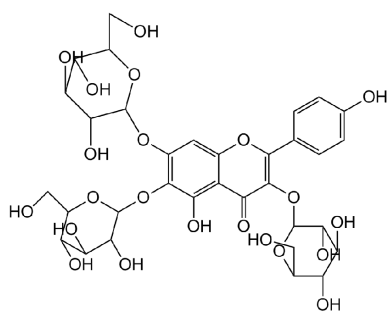

6-hydroxyl kaempferol-3,6,7-tri-glucoside

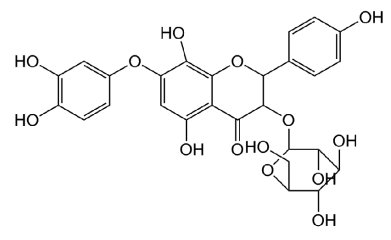

6-hydroxyl kaempferol-3-O-glucoside<smiles>O=C(O)C1Cc2cccc3c2C(=Cc2ccc(O)c(O)c2O3)C1=O</smiles>

Salvianolic acid G (m.w. 340)

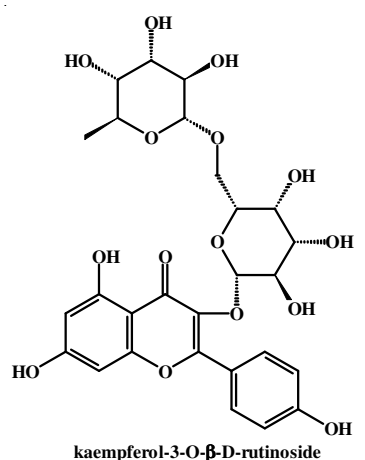

Fig. 1. Carbon skeletons of major compounds in Danhong injection is of great significance to develop a more reliable analytical method to characterize as much as chemical compositions from the view of quality control. Nowadays, it is promising that HPLC coupled with MS has been proved to be a very useful tool applied to the characterization of constituents in $\mathrm{TCMs}^{14}$. The present study aimed to develop the HPLC-UV fingerprint of Danhong injection and to characterize its major active constituents based on mass spectra. The fingerprint model could be used to accurately reflect the quality and guarantee clinical efficacy of Danhong injection. Metabolic HPLC fingerprint profiles were developed after intravenous administration of Danhong injection in rats at different time intervals in order to clarify the mechanism of clinical efficacy of Danhong injection. A chemical fingerprint combined with metabolic fingerprint would be helpful to recognize the differences between the in vitro and in vivo constituents in Danhong injection. This would be potential useful in establishing a suitable quality control model for Danhong injection.

\section{EXPERIMENTAL}

Acetonitrile (HPLC grade) was purchased from Honeywell (Muskegon, MI, USA). Glacial acetic acid and ethyl acetate were of analytical grade from Mallinckrodt Baker (Phillipsburg, NJ, USA). The standards of protocatechuic aldehyde, hydroxysafflor yellow A were purchased from the National Institute for the Control of Biological and Pharmaceutical Drugs (China). Salvianolic acid B and sodium danshensu were purchased from Guizhou Dida technology/Kexiang biological Co. Ltd. Rosmarinic acid was a gift from anesthesia department of Xijing Hospital. The deionized water was from Millipore water purification system (Milford, A, USA) and filtered with $0.45 \mu \mathrm{m}$ membrane. Danhong injection and its raw materials were provided by Shandong Buchang Pharmacy Co. Ltd. Batch numbers of Danhong injection are 101217, 110929, 110641, 110937, 110938, 110955, 110936, 110935, 110934, 110933, respectively. Pentobarbital sodium was obtained from Department of Physiology of Fourth Military Medical University. Heparin sodium was from shaanxi Biochemical Biotechnology Co. Ltd. Pentobarbital sodium and Heparin sodium were each dissolved in physiological saline to obtain the concentration of $50 \mathrm{mg} / \mathrm{mL}$ and $200 \mu \mathrm{g} / \mathrm{mL}$, respectively.

HPLC instrumentation and chromatographic conditions: An Agilent 1100 series system (Agilent Corporation) with diode-array detector was used for the chromatographic analysis. All HPLC profiles were carried on an Agilent Zobax Extend $\mathrm{C}_{18}$ reserved-phase analytical column $(250 \mathrm{~mm} \times 4.6$ $\mathrm{mm}, 5 \mu \mathrm{m}$, Agilent Corporation). The mobile phases included A (acetonitrile) and B (1\% glacial acetic acid aqueous solution) with gradient elution (0-10 $\mathrm{min}, 8-10 \% \mathrm{~A} ; 10-20 \mathrm{~min}, 10-$ $17 \%$ A; 20-30 min, 17-22\% A; 30-65 min, 22-30\% A; 65-80 min, $22-30 \% \mathrm{~A})$. There was a $15 \mathrm{~min}$ re-equilibration between individual runs to recover initial conditions. The column temperature was maintained at $25^{\circ} \mathrm{C}$. The detection wavelength was $280 \mathrm{~nm}$. The flow rate of the mobile phase was $1 \mathrm{~mL} / \mathrm{min}$ and the injection volume was $20 \mu \mathrm{L}$. In order to adapt to the flow rate to requirements of the ESI-MS, the flow rate was reduced by a split valve at that ratio of $1: 4$. Aliquots of $20 \mu \mathrm{L}$ were injected into the HPLC-MS. 

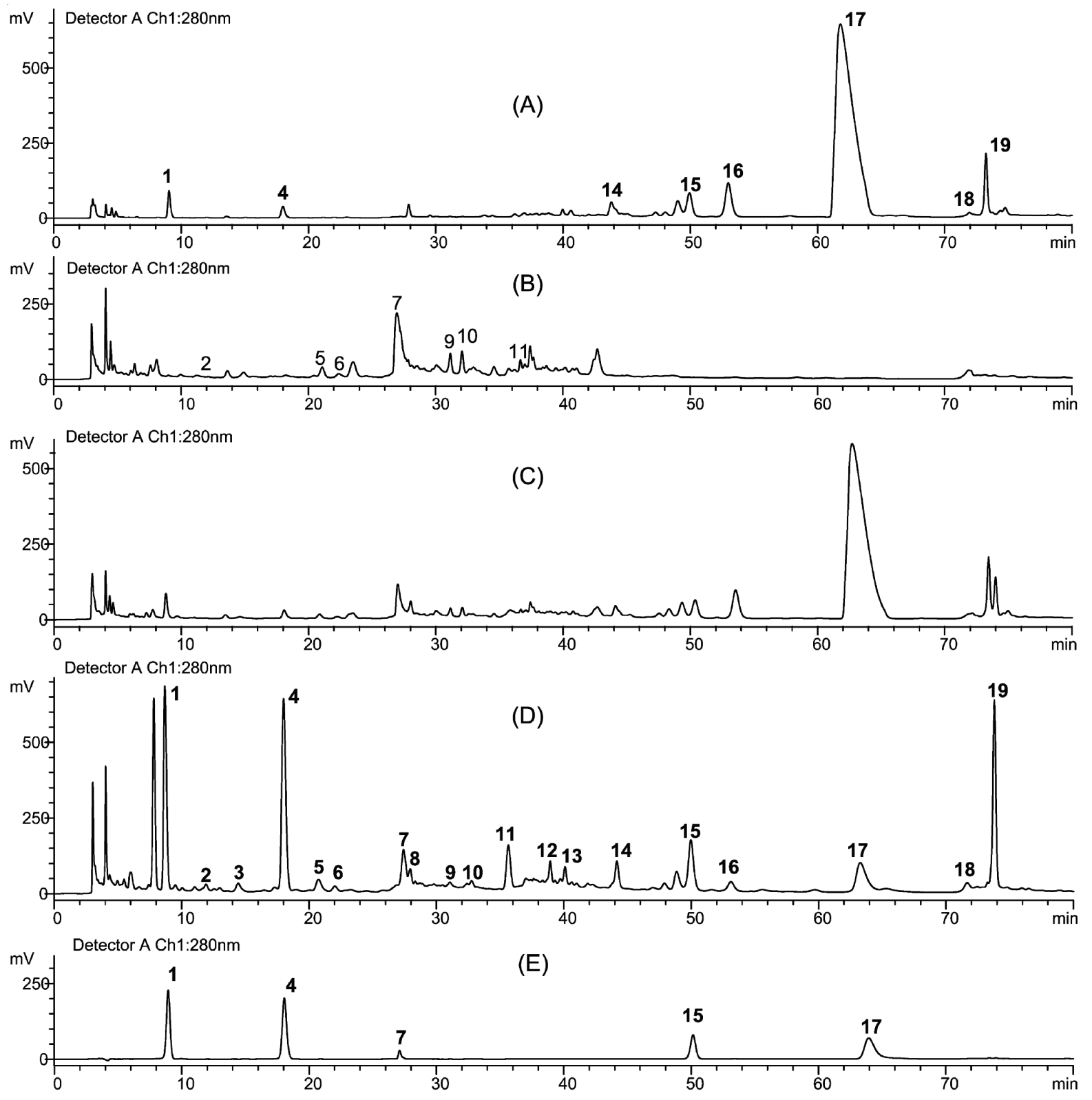

Fig. 2. HPLC-UV profiles of Radix Salviae Miltiorrhizae (A), Flos carthami (B), raw material of Danhong injection (C), Danhong injection (D) and standards (E). Peaks of standards represented: 1, danshensu; 4, protocatechuic aldehyde; 7, hydrosafflor yellow A; 15, rosmarinic acid; 17, salvianolic acid B

HPLC-MS was performed with an Agilent 1100 Series HPLC SCIEX QSTAR MASS. The HPLC conditions were the same as above. The mass spectra were recorded using ESI in both negative and positive mode. The ESI conditions were as follows: capillary voltage $=3500 \mathrm{~V}$, drying gas $=10 \mathrm{~L} / \mathrm{min}$, nebulizer gas $=45 \mathrm{psi}$, gas temp $=350{ }^{\circ} \mathrm{C}$, nozzle voltage $=$ $500 \mathrm{~V}$, mass range $=50-1500 \mathrm{~m} / \mathrm{z}$.

Animal and biological sample collection: Twelve male Sprague-Dawley (SD) rats (220-300 g body weight) were procured from the Laboratory Animal Center of the FMMU (Xi,an, Shaanxi). They were fasted for $24 \mathrm{~h}$ with free access to physiological saline before intravenous administration of Danhong injection. The rats were divided into four groups, with three in one group. After 5 min intravenous administration of physiological saline ( $2 \mathrm{~mL} / \mathrm{kg}$, body weight), three rats were anaesthetized by pentobarbital sodium and blank blood was collected from abdominal artery in clean heparinized glass tubes. Blank blood was centrifuged at $3500 \mathrm{rpm}$ to separate blank plasma. Danhong injections were administered to three groups of rats ( $2 \mathrm{~mL} / \mathrm{kg}$, body weight) by intravenous administration. Blood contained the chemical constituents from Danhong injection was collected as above workup after 5, 10 and 30 min of administration. Blank plasma and the corresponding plasma contained chemical constituents from Danhong injection were obtained. Plasma was frozen at $-20^{\circ} \mathrm{C}$ until analysis.

Sample preparation: Danhong injection was filtrated through $0.45 \mu \mathrm{m}$ filter for HPLC-UV and HPLC-MS analysis. The total phenolic acids in Radix Salviae miltiorrhizae and hydroxysafflor yellow A in Flos carthami were obtained by following extraction methods (these methods were provided by Shandong Buchang Pharmacy Co. Ltd. which manufactured Danhong injection). The powder of Radix Salviae miltiorrhizae $(5.0 \mathrm{~g})$ immersed in $40 \mathrm{~mL}$ deionized water and 
extracted under thermal reflux at a constant temperature of $102{ }^{\circ} \mathrm{C}$ for $1 \mathrm{~h}$ in oil bath (model HH-4, Guohua Electric Co., Jiangsu, China). The extraction process was repeated twice. At last, the extracts were combined, filtered while hot and evaporated on a vacuum concentrator system (Rongsheng, Shanghai, China) and then diluted to volume with deionized water in a $25 \mathrm{~mL}$ volumetric flask. Flos carthami $(5 \mathrm{~g})$ were immersed in $60 \mathrm{~mL}$ deionized water and extracted with the same method as Radix Salviae miltiorrhizae. The obtained solutions were filtered through $0.45 \mu \mathrm{m}$ filter for HPLC-UV analysis. The powder of Radix Salviae miltiorrhizae $(6 \mathrm{~g})$ and Flos carthami ( $2 \mathrm{~g}$ ) were mixed together in $64 \mathrm{~mL}$ deionized water and extracted also through the above process to gain the mixed raw material solution for HPLC-UV analysis.

The plasma sample $(1000 \mu \mathrm{L})$ was adjusted to $\mathrm{pH} 3$ by hydrochloric acid and extracted with EtOAc at the ratio of 1-2 (v/v) twice. Extraction was performed by vortex, mixing the tubes for $2 \mathrm{~min}$, followed by centrifugation at $3500 \mathrm{rpm}$ for $10 \mathrm{~min}$. The EtOAc fractions were collected into a glass tube and evaporated to dryness under a stream of nitrogen at $30^{\circ} \mathrm{C}$ water bath. The residue was dissolved in $400 \mu \mathrm{L}$ methanol and then filtered through a $0.45 \mu \mathrm{m}$ filter for HPLC-UV and HPLC-MS analysis.

A standard stock solution containing the 5 components (protocatechuic aldehyde $208.5 \mu \mathrm{g} / \mathrm{mL}$, hydroxysafflor yellow A $24.0 \mu \mathrm{g} / \mathrm{mL}$, rosmarinic acid $208.5 \mu \mathrm{g} / \mathrm{mL}$, salvianolic acid B $728.7 \mu \mathrm{g} / \mathrm{mL}$, sodium danshensu $1000.0 \mu \mathrm{g} / \mathrm{mL}$ ) was prepared in $50 \%$ methanol aqueous solution and stored away from light at $4{ }^{\circ} \mathrm{C}$.

\section{RESULTS AND DISCUSSION}

HPLC-UV fingerprint analysis of Danhong injection and the raw materials: Under the established HPLC conditions, HPLC profiles of Danhong injection and its corresponding raw materials were used to produce Danhong injection were analyzed. The HPLC-UV profiles of Danhong injection and the raw materials were presented in Fig. 3A-D. The constituents in Danhong injection and the raw materials were well separated under the established HPLC conditions. Danshensu, protocatechuic aldehyde, hydroxysafflor yellow A, rosmarinic acid and salvianolic acid B were recognized by comparing the retention times and UV spectra with their standards. The chromatograms of the five standard compounds were shown in Fig. 3E. Comparing the HPLC chromatograms (Fig. 3d and c) can found that the fingerprint profile of Danhong injection was greatly different from that of the raw materials. Danshensu, protocatechuic aldehyde, rosmarinic acid and salvianolic acid B were the main constituents in Danhong injection. However, the main constituent of Radix Salviae Miltiorrhizae was salvianolic B and hydroxysafflor yellow A was the main constitutent of Flos carthami. This indicated that the quantity of constituents had big changes during the preparation of Danhong injection. Salvianolic B was clearly decomposed and produced other phenolic acids. Hydroxysafflor yellow A was also decomposed to other compounds. Through comparing the HPLC-UV profiles of raw materials with Danhong injection (Fig. 3A, B and D), we could find that peak $1,4,14,15,16,17,18,19$ came from
Radix Salviae Miltiorrhizae and peak 2, 3, 5, 6,7, 9, 10, 11 from Flos carthami.

Methods validation: HPLC fingerprint determination is usually different from general assaying method. The authentication and identification of a drug and its products can be accurately performed using the chromatographic fingerprints, even if batches or concentrations varied among samples ${ }^{15}$.

To obtain a stable and repeatable chromatographic fingerprint of Danhong injection for quality control, the methods validation of HPLC fingerprint analysis was performed on the basis of the retention time and peak area. The sample solution of Danhong injection was successively injected into HPLC system for six times. The precision of relative retention times (RRT) and relative peak areas (RPA) of all peaks were not exceeding 3.59 and $3.74 \%$, respectively. To analyze the similarity between the 10 batches of Danhong injection, Similarity Evaluation System for Chromatographic Fingerprint of Traditional Chinese Medicine (Version 2004 A) was used ${ }^{16}$. The similarities of chromatograms of 10 batches comparing with the reference fingerprint was developed with the median of all chromatograms (Table-1). The stability test of sample solution was performed for $24 \mathrm{~h}$. The RSDs of relative retention time and relative peak area were less than 1.94 and $3.64 \%$, respectively. The results indicated that the method was valid and applicable for sample analysis. To establish the representative chromatographic fingerprint, 10 batches of Danhong injection were analyzed under the established HPLC-UV method. Peaks that appeared in all HPLC profiles of the 10 batches were assigned as "common peak" which represented the characteristics of Danhong injection. There were 20 "common peaks" in HPLC fingerprint (Fig. 4). The whole HPLC profile of the relative retention time and relative peak area of common peaks of the 20 "common peaks" (Tables 2 and 3) could be used to characterize the Danhong injection and assess the consistency from batch to batch.

TABLE-1

SIMILARITIES OF CHROMATOGRAMS OF 10 BATCHES

\begin{tabular}{cc|cc} 
No. & Similarities & No. & Similarities \\
\hline S1 & 0.978 & S6 & 0.982 \\
S2 & 0.937 & S7 & 0.977 \\
S3 & 0.944 & S8 & 0.977 \\
S4 & 0.984 & S9 & 0.963 \\
S5 & 0.981 & S10 & 0.949 \\
\hline
\end{tabular}

HPLC-MS analysis of Danhong injection: Danhong injection was analyzed by HPLC-MS technique to identify the structures of main constituents. ESI in both negative and positive mode were tried. The results showed that ESI in negative mode was sensitive to phenolic acids and ESI in positive mode was sensitive to glucosides. Major constituents in Danhong injection were well detected. Through carefully studying on the mass spectra of the compounds and comparing with the data of the standards and references, 19 common peaks in Danhong injection were designated and identified (Table4). They were danshensu (peak 1), quercertin (peak 2), 6hydroxyl kaempferol-3,6-di-O- $\beta$-glucoside (peak 3 ), protocatechuic aldehyde (peak 4), 6-hydroxyl kaempferol-3,6,7tri-O- $\beta$-glucoside (peak 5), 6-hydroxyl kaempferol-3-O- $\beta$-D- 

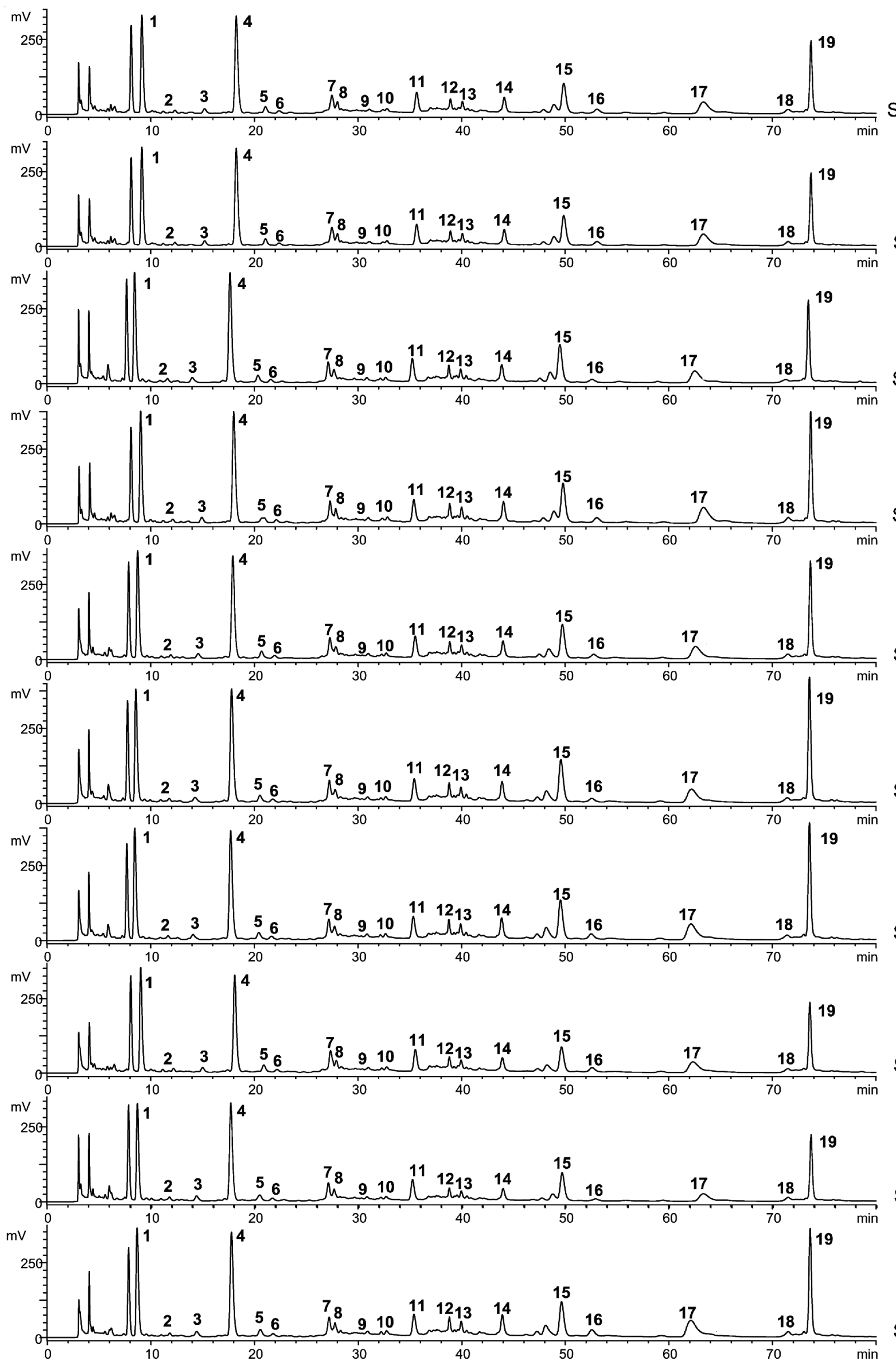

Fig. 3. HPLC fingerprint of 10 batches of Danhong injection marked peaks were common peaks of Danhong injection (S1-S10, respectively, represented 10 batches of Danhong injection: 101217, 110929, 110641, 110937, 110938, 110955, 110936, 110935, 110934, 110933) 


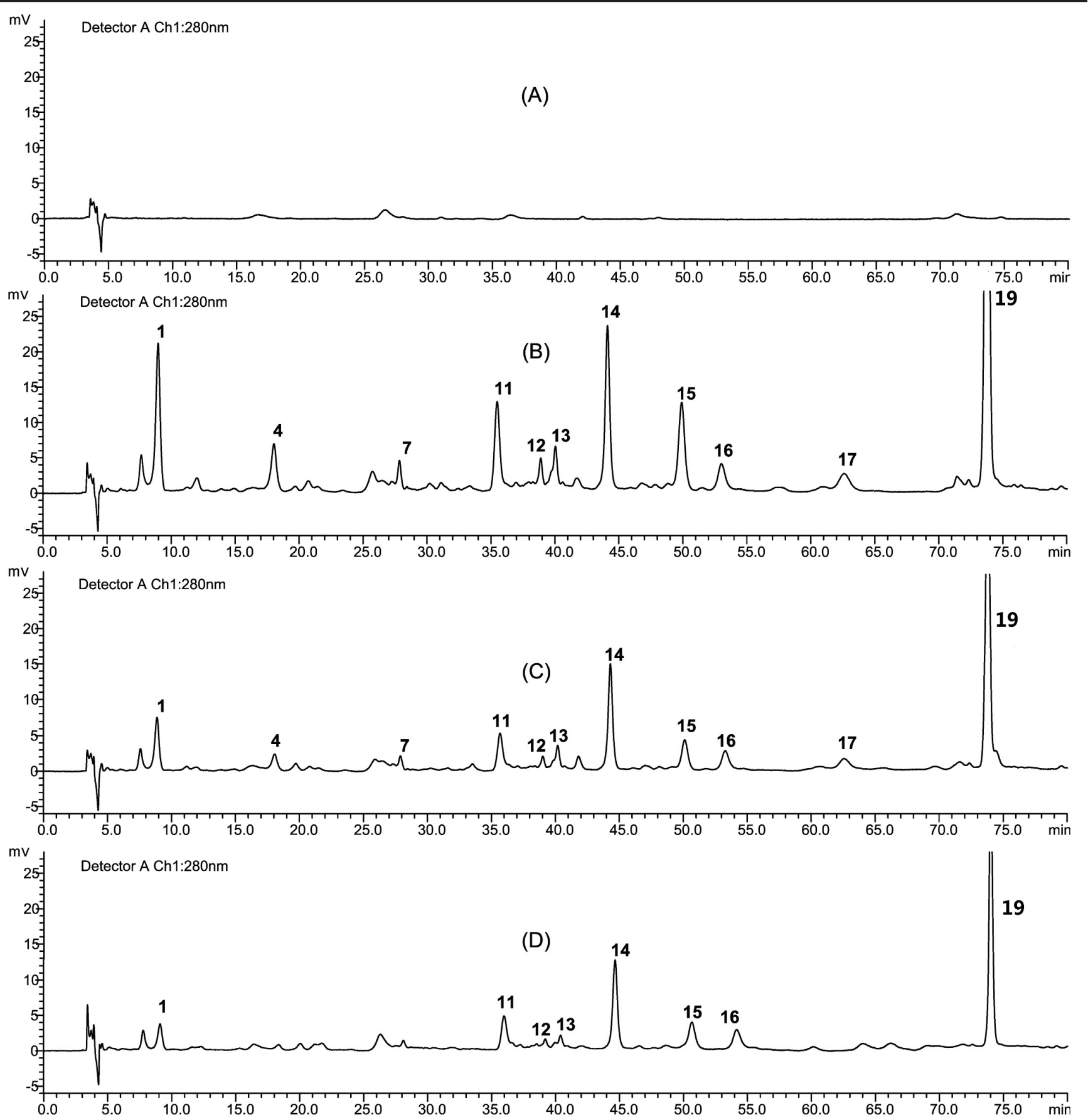

Fig. 4. HPLC profiles of blank rat's plasma (A) and plasma after 5 min (B), 10 min (C) and 30 min (D) administration, respectively. Peak number was consistent with the common peak number of Danhong injection

glucoside (peak 6), hydroxysafflor yellow A (peak 7), kaempferol-3-O- $\beta$-rutinoside (peak 8), roseoside (peak 9), carthamin or isocarthamidin (peak 10), caffeic acid (peak 11), salvianolic acid H (peak 12), salvianolic acid G (peak 13), salvianolic acid D (peak 14), rosmarinic acid (peak 15), lithospermic acid (peak 16), salvianolic acid B (peak 17), salvianolic acid C (peak 18), salvianolic acid A (peak 19) ${ }^{17-21}$, respectively.

Metabolic fingerprint analysis of Danhong injection: In order to clarify the active constituents which are responsible for the pharmacological action and guarantee the clinical efficacy of Danhong injection, it is necessary to know the metabolic changes and in vivo chemical constituents profile in biological systems. Therefore, rat plasmas after intravenous administration of Danhong injection at different time intervals, were analyzed by the same HPLC-UV method used for Danhong injection. The HPLC profiles were shown in Fig. 5. A comparision of the HPLC profiles from blank plasma showed that there was no interference found around the main active constituents of Danhong injection. It could be considered that the established plasma preparation method and HPLC analytical method for Danhong injection were suitable for the analysis of active constituents in rat blood. In order to identify the main constituents in rat plasma, samples obtained 5 min after intravenous administration of Danhong injection were analyzed using the established HPLC-MS method. 


\begin{tabular}{|c|c|c|c|c|c|c|c|c|c|c|c|}
\hline \multirow{3}{*}{$\begin{array}{l}\text { Common } \\
\text { peak No. }\end{array}$} & \multicolumn{10}{|c|}{$\begin{array}{c}\text { TABLE-2 } \\
\text { RELATIVE RETENTION TIME (RRT) IN HPLC FINGERPRINTS OF MEDICINAL DANHONG INJECTION }\end{array}$} & \multirow{3}{*}{$\operatorname{RSD}(\%)$} \\
\hline & \multicolumn{10}{|c|}{ Sample number } & \\
\hline & S1 & $\mathrm{S} 2$ & S3 & S4 & S5 & S6 & S7 & S8 & S9 & $\mathrm{S} 10$ & \\
\hline 1 & 0.49 & 0.50 & 0.48 & 0.48 & 0.48 & 0.49 & 0.50 & 0.49 & 0.50 & 0.48 & 0.93 \\
\hline 2 & 0.66 & 0.67 & 0.66 & 0.66 & 0.66 & 0.67 & 0.67 & 0.66 & 0.68 & 0.66 & 0.64 \\
\hline 3 & 0.81 & 0.83 & 0.80 & 0.80 & 0.79 & 0.81 & 0.83 & 0.81 & 0.83 & 0.79 & 1.45 \\
\hline 4 & 1.00 & 1.00 & 1.00 & 1.00 & 1.00 & 1.00 & 1.00 & 1.00 & 1.00 & 1.00 & 0.00 \\
\hline 5 & 1.16 & 1.15 & 1.15 & 1.15 & 1.15 & 1.15 & 1.16 & 1.15 & 1.15 & 1.15 & 0.29 \\
\hline 6 & 1.23 & 1.23 & 1.22 & 1.22 & 1.22 & 1.22 & 1.23 & 1.22 & 1.23 & 1.22 & 0.22 \\
\hline 7 & 1.53 & 1.51 & 1.53 & 1.53 & 1.53 & 1.52 & 1.52 & 1.53 & 1.51 & 1.54 & 1.05 \\
\hline 8 & 1.56 & 1.54 & 1.56 & 1.56 & 1.57 & 1.55 & 1.55 & 1.56 & 1.54 & 1.57 & 1.12 \\
\hline 9 & 1.74 & 1.71 & 1.74 & 1.74 & 1.74 & 1.73 & 1.72 & 1.73 & 1.70 & 1.75 & 1.44 \\
\hline 10 & 1.84 & 1.81 & 1.84 & 1.84 & 1.84 & 1.83 & 1.82 & 1.84 & 1.80 & 1.85 & 1.67 \\
\hline 11 & 1.99 & 1.96 & 1.99 & 1.99 & 1.99 & 1.98 & 1.97 & 1.98 & 1.95 & 2.00 & 1.53 \\
\hline 12 & 2.18 & 2.14 & 2.18 & 2.19 & 2.19 & 2.17 & 2.16 & 2.18 & 2.13 & 2.20 & 2.06 \\
\hline 13 & 2.25 & 2.21 & 2.24 & 2.25 & 2.25 & 2.23 & 2.22 & 2.24 & 2.20 & 2.26 & 2.11 \\
\hline 14 & 2.47 & 2.43 & 2.47 & 2.47 & 2.48 & 2.46 & 2.45 & 2.47 & 2.42 & 2.49 & 2.23 \\
\hline 15 & 2.66 & 2.61 & 2.66 & 2.67 & 2.67 & 2.65 & 2.66 & 2.67 & 2.62 & 2.69 & 2.33 \\
\hline 16 & 2.96 & 2.90 & 2.95 & 2.97 & 2.96 & 2.94 & 2.95 & 2.96 & 2.91 & 2.98 & 2.44 \\
\hline 17 & 3.49 & 3.44 & 3.49 & 3.51 & 3.51 & 3.49 & 3.52 & 3.52 & 3.47 & 3.54 & 2.82 \\
\hline 18 & 4.02 & 3.95 & 4.01 & 4.02 & 4.03 & 3.99 & 3.97 & 4.01 & 3.92 & 4.04 & 3.94 \\
\hline 19 & 4.14 & 4.07 & 4.13 & 4.15 & 4.15 & 4.11 & 4.09 & 4.13 & 4.04 & 4.16 & 4.03 \\
\hline
\end{tabular}

TABLE-3

RELATIVE AREAS OF THE COMMON PEAKS IN HPLC FINGERPRINTS OF DANHONG INJECTION

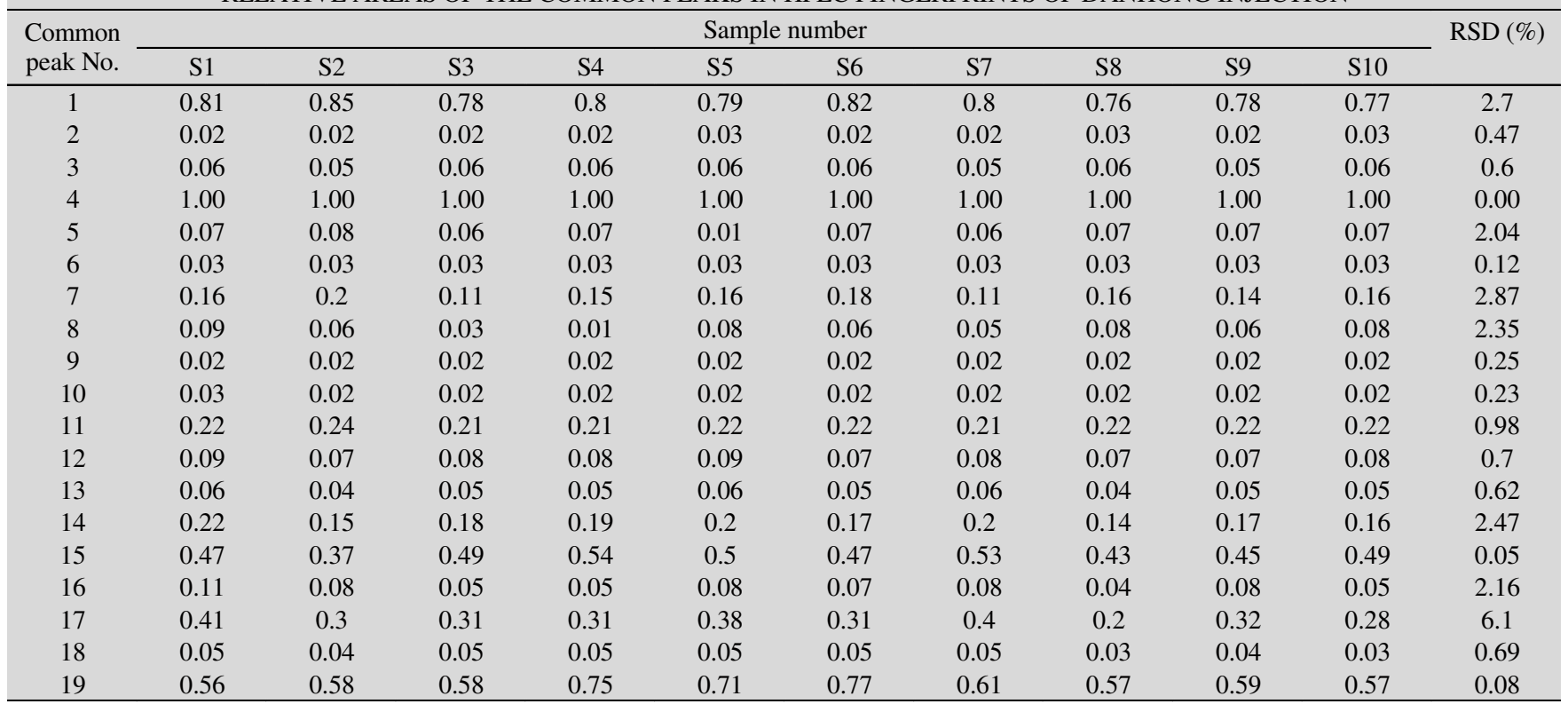

Phenolic acids and hydroxysafflor yellow A in rat plasma were also well separated and detected. The HPLC analytical results showed that the major phenolic acids and hydroxysafflor yellow A of Danhong injection all appeared in rat plasma, but the ratio of each constituent was greatly different from that in Danhong injection. This could be due to the differences in binding affinity of the phenolic acids and hydroxysafflor yellow A with plasma protein or to the difference in metabolic rate in blood. Danshensu, protocatechuic aldehyde, salvianolic acid A, B, D, rosmarinic acid, lithospermic acid, hydroxysafflor yellow A and caffeic acid were the major constituents in plasma at 5 min after administration. With the administration time prolonged, danshensu, protocatechuic aldehyde, hydroxysafflor yellow A, rosmarinic acid and salvianolic acid B reduced quickly, whereas salvianolic D, lithospermic acid, salvianolic
A and caffeic acid decreased slowly. Previous studies have demonstrated that danshensu, protocatechuic aldehyde, rosmarinic acid, salvianolic acid B and hydroxysafflor yellow A were active constituents in Danhong injection ${ }^{11,22}$. The pharmacokinetic profiles showed that distribution of danshensu was fast $^{23}$. It could be explained that danshensu distribute to various organs and generate pharmacological activity quickly. Salvianolic acid $\mathrm{B}$ decomposed to danshensu and salvianolic $\mathrm{A}^{24}$ and danshensu reduced much quicker than salvianolic A. It is suggested that danshensu has more potential to be the active constituent than salvianolic A. Therefore, danshensu, protocatechuic aldehyde, rosmarinic acid, salvianolic acid B and hydroxysafflor yellow A which reduced remarkably might be closely related to the pharmacological activity of Danhong injection. Furthermore, constituents which decreased quickly, 


\begin{tabular}{|c|c|c|c|c|}
\hline \multicolumn{5}{|c|}{$\begin{array}{c}\text { TABLE-4 } \\
\text { HPLC-MS DATA AND IDENTIFICATION FOR DANHONG INJECTION }\end{array}$} \\
\hline $\begin{array}{l}\text { Common } \\
\text { peak No. }\end{array}$ & $\begin{array}{l}\mathrm{TR} \\
(\mathrm{min})\end{array}$ & n.w. & ESI-MS (m/z) & Identification \\
\hline 1 & 8.72 & 198 & $197[\mathrm{M}-\mathrm{H}]^{-}, 179\left[\mathrm{M}-\mathrm{H}-\mathrm{H}_{2} \mathrm{O}\right]^{-}, 394[2 \mathrm{M}-\mathrm{H}]^{-}$ & Danshensu \\
\hline 2 & 11.89 & 302 & $301[\mathrm{M}-\mathrm{H}]^{-}, 283\left[\mathrm{M}-\mathrm{H}-\mathrm{H}_{2} \mathrm{O}\right]^{-}$ & Quercertin \\
\hline 3 & 14.50 & 624 & $625[\mathrm{M}-\mathrm{H}]^{-}, 463[\mathrm{M}-\mathrm{H}-\mathrm{glu}]^{-}, 301[\mathrm{M}-\mathrm{H}-\mathrm{glu}-\mathrm{glu}]^{-}$ & 6-Hydroxyl kaempferol-3,6-di-O- $\beta$-D-glucoside \\
\hline 4 & 17.88 & 138 & $137[\mathrm{M}-\mathrm{H}]^{-}$ & Protocatechuic aldehyde \\
\hline 5 & 20.65 & 788 & $\begin{array}{l}789[\mathrm{M}+\mathrm{H}]^{+}, 627[\mathrm{M}+\mathrm{H}-\mathrm{glu}]^{+} 456[\mathrm{M}+\mathrm{H}-\mathrm{glu}-\mathrm{glu}]^{+} \\
303[\mathrm{M}+\mathrm{H}-\mathrm{glu}-\mathrm{glu}-\mathrm{glu}]^{+}\end{array}$ & 6-Hydroxyl kaempferol-3,6,7-tri-O- $\beta$-D-glucoside \\
\hline 6 & 21.89 & 466 & $465[\mathrm{M}+1]^{+}, 303[\mathrm{M}+\mathrm{H}-\mathrm{glu}]^{+}$ & 6-Hydroxyl kaempferol-3-O- $\beta$-D-glucoside \\
\hline 7 & 27.26 & 612 & $611[\mathrm{M}-\mathrm{H}]^{-}$ & Hydroxysafflor yellow A \\
\hline 8 & 27.81 & 594 & $595[\mathrm{M}+1]^{+}, 287[\mathrm{M}+\mathrm{H}-\text { rutin }]^{+}$ & Kaempferol-3-O- $\beta$-rutinoside \\
\hline 9 & 30.94 & 386 & $385[\mathrm{M}-\mathrm{H}]^{-}, 431[\mathrm{M}-\mathrm{H}+\mathrm{HCOOH}]^{-}, 223[\mathrm{M}-\mathrm{H}-\mathrm{glu}]^{-}$ & Roseoside \\
\hline 10 & 32.73 & 448 & $447[\mathrm{M}-\mathrm{H}]^{-}, 287[\mathrm{M}-\mathrm{H}-\mathrm{glu}]^{-}$ & Carthamin/isocarthamidin \\
\hline 11 & 35.42 & 180 & $179[\mathrm{M}-\mathrm{H}]^{-}, 135[\mathrm{M}-\mathrm{H}-\mathrm{COO}]^{-}$ & Caffeic acid \\
\hline 12 & 38.81 & 538 & 537[M-H] $]^{-}, 339[\mathrm{M}-\mathrm{H}-198]^{-}, 295[\mathrm{M}-\mathrm{H}-\mathrm{COO}-198]^{-}$ & Salvianolic acid $\mathrm{H}$ \\
\hline 13 & 39.95 & 340 & $339[\mathrm{M}-\mathrm{H}]^{-}, 141[\mathrm{M}-\mathrm{H}-198]^{-}$ & Salvianolic acid G \\
\hline 14 & 43.95 & 418 & $417[\mathrm{M}-\mathrm{H}]^{-}, 373[\mathrm{M}-\mathrm{H}-\mathrm{COO}]^{-}, 157[\mathrm{M}-\mathrm{H}-\mathrm{COO}-198]^{-}$ & Salvianolic acid D \\
\hline 15 & 49.64 & 360 & $359[\mathrm{M}-\mathrm{H}]^{-}, 161[\mathrm{M}-\mathrm{H}-198]^{-}, 719[2 \mathrm{M}-\mathrm{H}]^{-}$ & Rosmarinic acid \\
\hline 16 & 52.70 & 538 & 537[M-H] $]^{-}, 339[\mathrm{M}-\mathrm{H}-198]^{-}, 295[\mathrm{M}-\mathrm{H}-\mathrm{COO}-198]^{-}$ & Lithospermic acid \\
\hline 17 & 62.56 & 718 & $717[\mathrm{M}-\mathrm{H}]^{-}, 519[\mathrm{M}-\mathrm{H}-198]^{-}, 320[\mathrm{M}-\mathrm{H}-198-198]^{-}$ & Salvianolic acid B \\
\hline 18 & 71.44 & 718 & $491[\mathrm{M}-\mathrm{H}]^{-}, 293[\mathrm{M}-\mathrm{H}-198]^{-}, 265[\mathrm{M}-\mathrm{H}-198-\mathrm{CO}]^{-}$ & Salvianolic acid C \\
\hline 19 & 73.61 & 494 & $493[\mathrm{M}-\mathrm{H}]^{-}, 295[\mathrm{M}-\mathrm{H}-198]^{-}$ & Salvianolic acid A \\
\hline
\end{tabular}

such as danshensu, protocatechuic aldehyde, rosmarinic acid, salvianolic acid B and hydroxysafflor yellow A, will be further investigated in the coming months using animal models of cardiovascular and cerebrovascular diseases.

\section{Conclusion}

From all these observations, it can be concluded that HPLC-UV and HPLC-MS techniques were used in chemical fingerprint and metabolic fingerprint analysis of Danhong injection and its raw materials. HPLC profiles of Danhong injection and their raw materials were established as their characteristic fingerprint which were employed to assess their consistency and difference. A chemical fingerprint of Danhong injection was established and 19 major constituents of Danhong injection were identified by comparing their mass spectra and retention behaviour with reference standards or literature data. Most of the major constituents in Danhong injection were detected and identified in rat plasma after intravenous administration. It was useful for better understanding the in vivo metabolism of Danhong injection. In addition, this study offered scientific data regarding the active constituents of Danhong injection and provided marked compounds, such as danshensu, protocatechuic aldehyde, rosmarinic acid, salvianolic acid B and hydroxysafflor yellow A, for preparation improvement and quality control of Danhong injection.

\section{ACKNOWLEDGEMENTS}

This study was supported by National Science Foundation of China (ADW, No. 81173514), Major National Drug Discovery (ADW, No. 2011ZXJ09202-13), Xijing Research Boosting Program (MMX, NO. XJZT10D02), National Natural Science Foundation of China (No. 81001673), Natural Science Foundation of Shaanxi Province, PR China (No. 2010JQ4018) the "13115" Technology Innovation Project of Shaanxi Province, P.R. China (No. 2010ZDKG-62).

\section{REFERENCES}

1. Y.X. Zhou, H.M. Lei, Y.H. Xu, L.X. Wei and X.F. Xu, Research Technology of Fingerprint of Chinese Traditional Medicine, Beijing (2002).

2. M. Gu, F. Ouyang and Z.G. Su, J. Chromatogr. A, 1022, 139 (2004).

3. Drug Administration Bureau of China, Requirements for Studying Fingerprint of Traditional Chinese Medicine Injections (Draft) (2000).

4. X.H. Fan, Y. Wang and Y.Y. Cheng, J. Pharm. Biomed. Anal., 40, 591 (2006).

5. L.F. Hu, S.P. Li, H. Cao, J.J. Liu, J.L. Gao, F.Q. Yang and Y.T. Wang, J. Pharm. Biomed. Anal., 42, 200 (2006).

6. Y.Z. Liang, P.S. Xie and K. Chan, J. Chromatogr. B, 812, 53 (2004).

7. P.S. Xie, Chromatography Fingerprint of Traditional Chinese Medicine, People's Medical Publishing House, Beijing, pp. 18-104 (2005).

8. J. Zh. Xu, J. Shen, Y.Y. Cheng and H.B. Qu, J. Zhejiang Univ. Sci. B, 9, 728 (2008).

9. J.L. Zhang, M. Cui, Y. He, H.L. Yu and D.A. Guo, J. Pharm. Biomed. Anal., 36, 1029 (2005).

10. Y. Liu, J. Yang and Q. Liu, J. Chin. Med. Mater, 28, 288 (2005).

11. H. He, X. Yang, M. Shi, X. Zeng, J. Yang, L. Wu and L. Li, J. Pharm. Pharmacol., 60, 115 (2008).

12. M. Sun, J.J. Zhang, J.Z. Shan, H. Zhang, C.Y. Jin, S. Xu and Y.L. Wang, Phytomedicine, 16, 683 (2009).

13. National Chinese Patent Medicines Standard Assembly, Medical, Heart Volumes, WS-11220(ZD-1220) (2002).

14. J. Jing, C.O. Chan, L. Xu, D. Jin, X. Cao, D.K. Mok, H.S. Parekh and S. Chen, J. Pharm. Biomed. Anal., 56, 830 (2011).

15. F. Gong, Y.Z. Liang, P.S. Xie and F.T. Chau, J. Chromatogr. A, 1002, 25 (2003).

16. Y. Chen, Y. Yan, M.Y. Xie, S.P. Nie, W. Liu, X.F. Gong and Y.X. Wang, J. Pharm. Biomed. Anal., 47, 469 (2008).

17. H.Y. Ling, X. Zh. Lu, Y.L. Zhao and Sh. W. Zhang, Nat. Prod. Res. Dev., 11, 75 (1999).

18. L. Fan, H.Y. Zhao, M. Xu, L. Zhou, H. Guo, J. Han, B.R. Wang and D.A. Guo, J. Chromatogr. A, 1216, 2063 (2009).

19. Y. Champavier, G. Comte, J. Vercauteren, D.P. Allais and A.J. Chulia, Phytochemistry, 50, 1219 (1999).

20. Y. Jin, Y.S. Xiao, F.F. Zhang, X.Y. Xue, Q. Xu and X.M. Liang, J. Pharm. Biomed. Anal., 46, 418 (2008).

21. Y.M. Liu, J.S. Yang and Q.H. Liu, J. Chin. Med. Mater., 28, 288 (2005).

22. M. Ruan, Y. Li, X. Li, J. Luo and L. Kong, J. Pharm. Biomed. Anal., 59, 184 (2012).

23. Y. Li, X. Li, L. Wang, Y. Li, Y. Xu and M. Xue, J. Pharm. Biomed. Anal., 44, 1106 (2007).

24. S.S. Liu, F. Han, H. Xu and K. Liu, Chin. Trad. Herbal Drugs, 2, 247 (2011). 